\title{
Conception and evaluation of a washable multimodal smart textile
}

\author{
Nils A. Lahmann ${ }^{1}$. . Ursula Müller-Werdan ${ }^{1}$ - Simone Kuntz ${ }^{1}$ Jürgen Klingehöfer-Noe ${ }^{2}$ - Fabian Jaenicke ${ }^{3}$. \\ Sandra Strube-Lahmann ${ }^{1}$
}

Received: 6 August 2021 / Accepted: 4 November 2021 / Published online: 11 November 2021

(c) The Author(s) 2021

\begin{abstract}
Smart textiles can support people with specific needs and diseases, such as diabetes or heart disease. Currently there are efforts to combine continuous mobile monitoring with other health-related conditions. On this basis, algorithms could be developed that can be used to detect unusual or critical conditions. A study was to investigate whether a previously developed washable Multi-Modal Smart Textile (MMST), based on inexpensive materials, would provide valid and reliable results with regard to the vital parameters of pulse, temperature and mobility. The measurement of the vital parameters was carried out with the developed prototype MMST as well as with validated devices. All electronics including the rechargeable NiMH has been washed more than 30 times with different methods and it remained fully functional. The intraclass correlation coefficients (ICC) for pulse (temperature) measurement ranged between 0.036 and 0.232 (0.077 and 0.817) depending on the activity of the tested individuals (standing, sitting, lying down, moving). Cohen's Kappa for the detection of the body position was 0.765 . For the parameter of pulse, the results indicated an insufficient derivation for both validity and reliability. Due to flaws in the methodology applied, the validly and reliably for the parameter of temperature could not be determined. Valid and reliable results were obtained for the parameter mobility/change of position. If the MMST (after modification of the prototype) achieves reliable results, there are many advantages for people giving and receiving care on a budget price, even in threatening emergency situations.
\end{abstract}

Keywords Smart textiles $\cdot$ Nursing $\cdot$ Sensors $\cdot$ Care $\cdot$ Pulse $\cdot$ Temperature $\cdot$ Body position

Nils A. Lahmann

nils.lahmann@charite.de

Ursula Müller-Werdan

ursula.mueller-werdan@charite.de

Simone Kuntz

simone.kuntz@charite.de

Jürgen Klingehöfer-Noe

Juergen.Klingelhoefer-Noe@homeinstead.de

Fabian Jaenicke

fabian.jaenicke@freyersiegel.de

Sandra Strube-Lahmann

sandra.strube-lahmann@charite.de

1 Geriatrics Research Group, Department of Geriatrics and Medical Gerontology, Charité - Universitätsmedizin Berlin, Corporate member of Freie Universität Berlin, Humboldt-Universität zu Berlin, and Berlin, Augustenburger Platz 1, 13353 Berlin, Germany

2 HISB Betreuungsdienste Berlin-Sued, Margaretenstraße 38, 12203 Berlin, Germany

3 Freyer \& Siegel, Elektronik GmbH \& Co. KG, Kastanienallee 19, 16567 Mühlenbeck, Germany

\section{Introduction}

Smart textiles were already mentioned in publications 20 to 25 years ago [1-4], but they have become more visible in scientific research in the last few years. For medical purposes, they can be distinguished between those with a more chemical functionality i.e. photothermal, $\mathrm{pH}$ analytic or antibacterial activities $[5,6]$ and others containing electrical / electronic properties [7]. Since there has been a rapid technological development in general in the past few years, smart wearable devices explicitly have gained enormous popularity in healthcare [8]. They have proven to support individuals with specific needs and diseases like diabetes [9], asthma [10] and the detection of heart rate arrhythmia [11].

Activity monitoring via mobile devices such as watches has become very popular in the so-called "lifestyle" sector. However, since the importance of activity and mobility for physical and mental health is well recognized, there have been recent efforts to combine continuous mobile monitoring with other health-related conditions, such as continuous 
glucose monitoring [12] or bowel status [13]. By combining different parameters such as vital signs, glucose monitoring and / or activity, it is possible to develop algorithms that could be used to recognize unusual or critical conditions, especially if these parameters are available in a continuous manner, as is done with highly dependent patients in intensive care. Even if individuals are not in such a critical health condition, but more or less dependent on care, continuous mobile monitoring may help to improve their treatment, i.e. by adapting their medication. Integrating these monitors into objects of daily use, such as clothes, has some promising advantages. First, clothes are worn close to the body, which is a prerequisite for the precise monitoring of vital signs. Second, it looks more natural to them and is therefore less stigmatizing and people with cognitive impairment do not tolerate additional "hang-ons" like wristbands or badges.

In the last decade, a variety of vital textiles has been developed that combine both a clothing and measuring functions of vital signs such as pulse rate, heart rate. Smart textiles could measure vital signs pulse rate, heart rate of human, mobility or temperature and developed for different scenarios for example health care workers, fire fighters, mineworkers or fitness trainers [14-16]. The possibilities in applications sound promising, but there is still further need for research and development in embedding microelectronics in textiles. Electronic components have to be reliable and robust, even if measurement signals are distorted in their usage behaviour. The textile surfaces used for Smart Textiles in general and Vital Textiles (Textiles measuring vital signs like pulse, temperature, breathing rate etc.) in particular must be stretchable, resistant to environmental factors, periodic maintenance such as washing and drying, as well as resistant to friction and movement over the long term [17]. This is very important if textiles are worn on body areas where people can sweat a lot like in fiber-optics sensors equipped smart socks [18]. This places much higher demands on the electronic components to be incorporated than in the manufacture and processing of classic electronics. Interesting approaches such as the smart textile base on fibre optics technology [19-21] were considered a-priori to be too sensitive for this purpose. The insufficient resistance of the electronic components to water and the washing additives is caused by the following problems: 1 . corrosion of metallic and conductive components, 2. thermal issues (washing, drying, defrosting temperature, exposure time), 3. mechanical issues (pressure, torsion, bending and buckling of contact points to electronic components through washing, draining and finishing treatments), and 4. chemical stress (alkalinity, oxidative attack by detergents/disinfectants). Zhou et al. achieved a decent washability for their developed single-layered ultra-soft smart textiles when they washed it several times 10 to $60 \mathrm{~min}$ at $30{ }^{\circ} \mathrm{C}$ [22]. Merino Wool/Nylon Polymer Nanocomposites show also some promising developments in this regard [23]. Some nanocomposite fibers of conjugated polymer nanoparticles and polyacrylonitrile showed efficient durability after a laundry test [5]. An e-textile's degradation performance should not only be measured through the change in conductivity, and electrical resistance [24] but also by the devices performance after washing $[25,26]$. If the electronic components are not embedded in elastomer material like polydimethylsiloxane (PDMS, silicone) [27], smart textiles are often designed as a modular system to separate the washable textile parts from the electronic components.

The aim of this study comprised different developments and tests. We tried to integrate into a textile:

1. washable treatment sensors for recording movement or localisation, measuring critical vital parameters (i. e. pulse, body temperature), detection of position (i.e. fall., impared mobility)

2. washable, treatment-resistant energy transfer and storage systems,

3. washable, treatment-resistant measuring technology for data transmission, which is inseparably linked to the garment textile.

Moreover, a mobile application for the presentation of the data had to be designed and the data, provided by the system, had to be tested. The contribution of the planned technological development was the elaboration of sensors and energy transfer and storage systems which are resistant to treatments such as washing and disinfection for the application nursing care. Finally it must be emphasized, that the overall aim was not to build an high-end medical device but to remain "affordable" for many care dependent people. For this purpose, the components to assemble the smart textile is based on rather established, commercial off-the-shelves technology and because the monitoring function of the smart textile should rather serve as a screening tool to detect rather gradual changes over a longer period of time, no absolute accuracy would be necessary. For that reason, that the installed electronic components can also be significantly cheaper.

\section{Material and methods}

\subsection{Apparatus}

The first potting tests that were carried out with various one- and two-component silicone rubbers did not yield satisfactory results. The materials used either did not create a water-impermeable connection at the interface to the cables or did not have the desired tear resistance (Fig. 1a). In order to achieve washability, the entire electronic assembly 

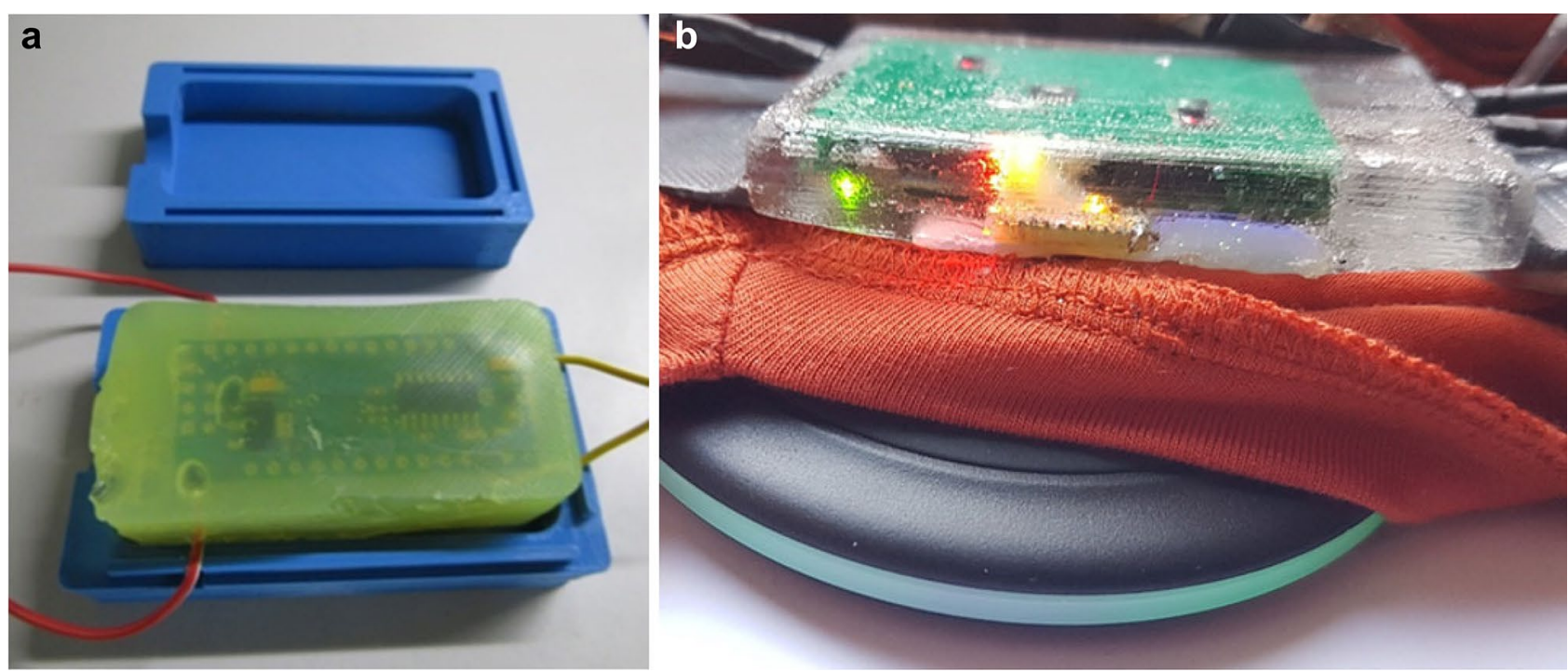

Fig. 1 a potting tests with silicone rubbers (not enough visibility) b Showed the in Wepuran VT 3402 encapsuled and washed battery unit (after 30 Washing processes) attached to the textile on the wireless charger while charging

was cast with Wepuran casting resin VT 3402 (From Company: Lackwerke Peters $\left.{ }^{\mathrm{TM}}\right)$. After the Wapuran was mixed according the proposed mixing ratio, it hardened-24 $\mathrm{h}$ at room temperature $\left(20-23,5^{\circ} \mathrm{C}\right)$ hardened for $4 \mathrm{~h}$ in warming cabinet at $45^{\circ} \mathrm{C}$, then again for $15.5 \mathrm{~h}$ at room temperature $\left(23.5-26.5^{\circ} \mathrm{C}\right)$, and again $8 \mathrm{~h}$ in warming cabinet at $45^{\circ} \mathrm{C}$. After another $24 \mathrm{~h}$ at room temperature $\left(23.5-26.5^{\circ} \mathrm{C}\right)$ it was removed from the plastic form. This enabled a watertight enclosure of the assembly, high tear resistance, rubberlike elasticity, temperature resistance up to at least $40{ }^{\circ} \mathrm{C}$ (washing mashine temperature) and transparency for visibility of the light of LED inside. A rechargeable $\mathrm{NiMH}$ battery (Varta 3/V500HTLSWC, three flat battery cells with a nominal voltage of $1.2 \mathrm{~V}$ in series (i.e. $3.6 \mathrm{~V}$ in total) and a Qi-compatible induction loop for charging the battery were integrated into the smart textiles (Fig. 1b). The battery can be charged via cable or wirelessly.

The energy-efficient microcontroller STM32-L151 by ST Microelectronics ${ }^{\mathrm{TM}}$ was selected for recording and processing the sensor data (Fig. 2). Low electricity usage was a factor taken into consideration when choosing the sensor-ICs. The data transmission close to the electronics was done via copper cables and wires. The connection of textile sensor technology with the copper cable was done with heat shrink self-solder butt splice connectors. We decided to use Photoplethysmography (PPG) to monitor the heart rate. Two diagonally arranged sensors (Maxim Integrated $^{\mathrm{TM}}$ MAX30102) were integrated on a platform to measure the heart rate. Preliminary tests have shown that two diagonally arranged pulse sensors suffice for a sufficiently accurate pulse measurement (Fig. 3). We used the infrared temperature sensor Melexis MLX90615 to measure the body surface temperature on the breast on the skin site. This sensor can measure the temperature even without direct skin contact at a distance of approx. $1 \mathrm{~cm}$ from the skin surface. In order to conduct the body position, a 3-axis accelerometer (NXP MMA8451Q) is included. The algorithm we used was the following (simplified description):

1. Every $200 \mathrm{~ms}$ : Calculation of the angle change of roll $\varphi$ (phi) pitch $\theta$ (theta) and yaw angle $\varepsilon$ (eta) from the acceleration data ax, ay, az of the acceleration sensor.

2. angle change is large enough:

If phi $>$ activity threshold- $>$ body upright.

If theta $>$ activity threshold- $>$ left lateral position.

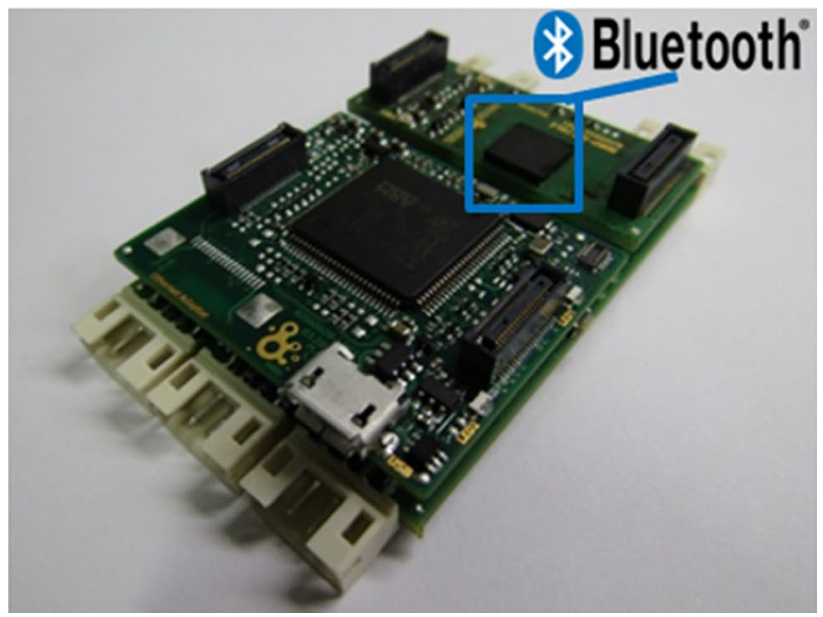

Fig. 2 shows the front (facing the textile) side of the in-built microelectronic system 


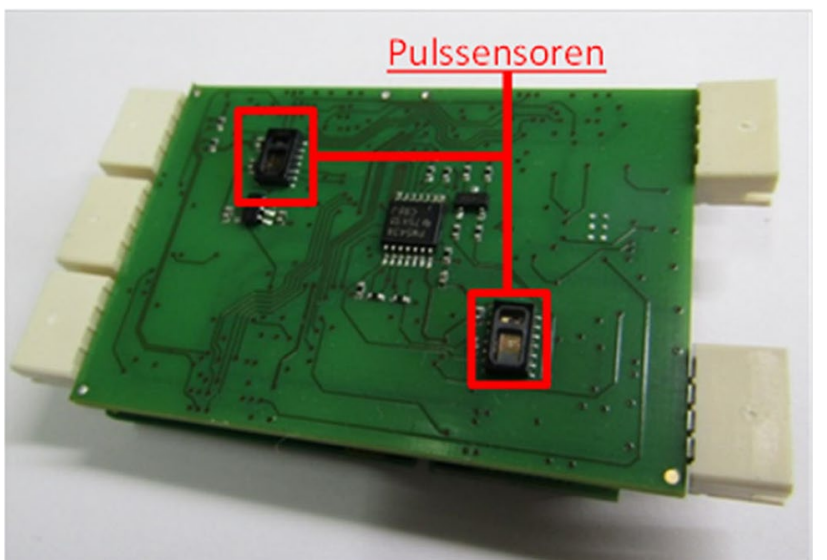

Fig. 3 shows and rear side (facing the body) (from left) of the in-built microelectronic system ([w x h x d] $4,9 \times 3.4 \times 0.5)$

If theta $<$ negative activity threshold- $>$ lateral position right.

If eta $>$ activity threshold- $>$ supine position.

3. Furthermore, there is a check of the time since the last change of movement. Only if this is greater than two seconds is a new position displayed. Otherwise, there would be continuous movement changes at certain "limit angles", for example.

The frequency of data recording was for pulse (raw data from photo diode): every $100 \mathrm{~ms}$, movement (change of acceleration): every $200 \mathrm{~ms}$, and temperature (I2C-sensor data): every $10 \mathrm{~s}$. To communicate with the Smartphone App we used a Cypress Semiconductor CYW20736S Bluetooth Low Energy System-in-Package module. This enables a Bluetooth connection to a smartphone. Data updating in the app for every parameter occurred only in case of a change, but not more than every second. The system can also store data on a micro SD card.

The hardware was integrated into a stretchable and conformable textile via a transparent encapsulate with Wepuran casting resin VT 3402 in such a way that clinical hygiene standards are met (Fig. 4a). A schematic description of the whole system is illustrated in Fig. 4b. In order to make the smart textile "wearable" it was integrated in a tailored home-made T-shirt. To find the best location for the device we conducted a workshop with elderly persons and nursing practitioners about the comfort and the handling of the device. The participants stated that a position on the front breast was regarded comfortable the most. We explained to the participants that this prototype has to be rather bulky and big for development purpose and that our aim is to miniaturise the electronic to a size of about $25 \times 25 \times 7 \mathrm{~mm}$ and less than $30 \mathrm{~g}$, once the proof of concept is satisfactory. The feedback from the participants was mostly that such a small size would most likely not disturb them. The exchangeable battery was housed in the integrated non-slip breast pocket of a shirt.

The data were shown as pictures, graphics and pure measurement values as an Android application for smartphones (Fig. 5). The patient can define individual standards and limit values for his or her vital parameters. In the app, the measured vital signs were displayed as a traffic light system. Normal values showed as a green background, deviating values with a yellow background and dangerous values as a red background. All typical sizes were manufactured but for the testing only the clothing sizes ( $\mathrm{S}$ and $\mathrm{M}$ ) were used because they fitted best to the participants.

\subsection{Washability testing}

The electronic module encapsulated with Wepuran VT 3402 (Fig. 1b) with soldered-on leads was used to carry out washing cycles at $40^{\circ}, 60^{\circ}$ and $95^{\circ}$ Celsius with and without fabric softener The washing mashine was a consumer model (Miele Navitronic W2888). Afterwards the analogue inputs were checked for optical damage. The functionality of the digital and analog inputs was checked with a small test arrangement. An integrated LED indicated 4 cases of functionality: Case 1: Slow flashing (1.6 s) OK; Case 2: Fast flashing (0.4 s), water has penetrated; Case 3: PWR LED only, cable from digital input defective; Case 4: No LED, electronics defective.

\subsection{Participants and study design}

We compared the vital signs of pulse and skin temperature of the developed Multi-Modal Smart Textile (MMST) prototype with a validated pulse oximeter (OxyTrue ${ }^{\circledR}$ A SMARTsatSpO2 monitor by bluepoint MEDICAL GmbH \& Co. KG) and a laser surface thermometer (Basetech 350C12 infraredthermometer Optik 12:1 -50 to $350{ }^{\circ} \mathrm{C}$ Pyrometer). The sensory measurement of mobility and body position was validated with the clinical view of experts.

The testing comprised two different phases. Phase 1 was to compare values of the MMST to values of an external measurement mentioned above to check the data for validity. Phase 2 was to continuously record data for some period of time to check for reliability. Both phases took place between May and July 2019 and were conducted in the laboratory of the Geriatrics Research Group in Berlin. In order to recruit suitable test persons, information materials (test person information, flyers, etc.) were handed out during public presentations of the project and we placed a public notice in our facilities to find participants for the study. We informed the participants about the study procedure and data protection. After giving their informed consent, the test persons received the prototype of the shirt. Empirical testing of 

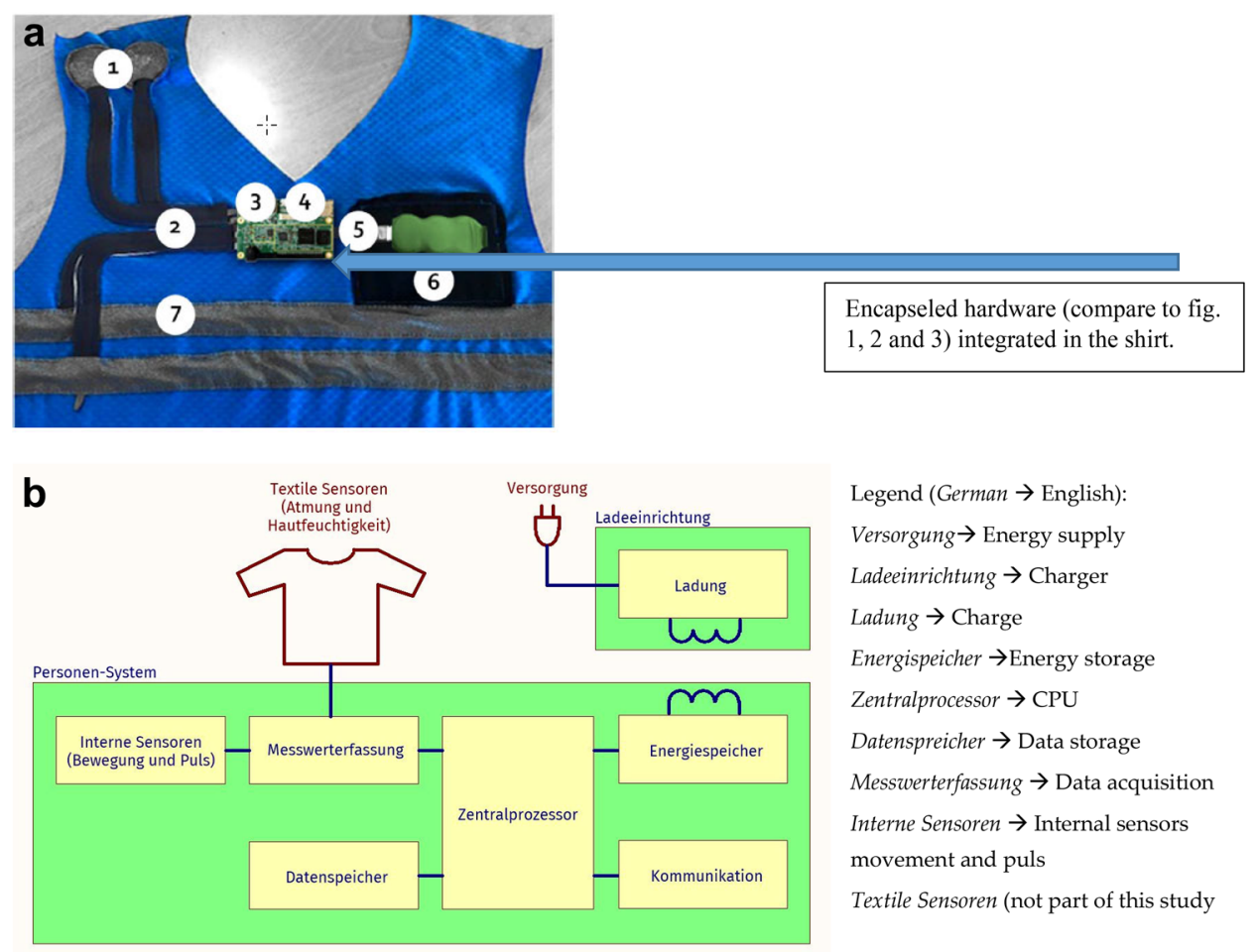

\author{
Legend (German $\rightarrow$ English): \\ Versorgung $\rightarrow$ Energy supply \\ Ladeeinrichtung $\rightarrow$ Charger \\ Ladung $\rightarrow$ Charge \\ Energispeicher $\rightarrow$ Energy storage \\ Zentralprocessor $\rightarrow \mathrm{CPU}$ \\ Datenspreicher $\rightarrow$ Data storage \\ Messwerterfassung $\rightarrow$ Data acquisition \\ Interne Sensoren $\rightarrow$ Internal sensors \\ movement and puls \\ Textile Sensoren (not part of this study
}

Fig. 4 a: Functional sample with integrated microelectronics ([w $\mathrm{x} \mathrm{h} \mathrm{x} \mathrm{d]} 6.9 \times 4.4 \times 1.1 \mathrm{~cm}$ ) and interior breast pocket to accommodate the battery (T-Shirt is inside out) The functional model consists of the assemblies shown in Fig. 4a. 1. skin moisture sensors made of electrically conductive, stretchable knitted or embroidered materials. ( $\rightarrow$ did not pass functionality test and was therefore excluded for the study) 2. waterproof, washable, flexible textile surface conductors $\AA$, which can also be directly contacted and encapsulated directly with the electronics, thus providing strain relief.3. processing unit in the form of microprocessor hardware. 4. sensors for movement and body position (update frequency $200 \mathrm{~ms}$ ), skin temperature (update frequency $10 \mathrm{~s}$ ) and pulse (update frequency $100 \mathrm{~ms}$ ). 5. area con-

different positions and situations (walk, sit, stand, lie down on different sides) of healthy test persons involved testing the data-gathering quality of the smart textiles regarding vital signs and activity data detectable by the sensors. In order to test the optimal position of the sensors on or in the textile, it was necessary for the subjects to wear the smart textile prototype for a short time.

The testing started with phase 1: First, the vital signs and movement were measured while the subject was resting. The same measurements were then taken just after the subject had exercised (going up and down 1-2 flights of stairs). Different body positions were tested, for example moving from a sitting to a standing position or while walking. At the same time, the measurements of the vital statistics and movements were taken with well established clinical devices (pulse), other external devices (temperature) and / or observation criteria (experts' observation of the movements). Skin temperature was measured using the laser surface thermometer ductor with battery connection and connection to the processing unit - located underneath the breast pocket. 6 . battery pocket with visualised battery. (later encapseled) 7. breathing sensor made of electrically conductive, stretchable knitted materials. ( $\rightarrow$ did not pass functionality test and was therefore excluded for the study) Legend (German $\rightarrow$ English): Versorgung $\rightarrow$ Energy supply Ladeeinrichtung $\rightarrow$ Charger Ladung $\rightarrow$ Charge Energispeicher $\rightarrow$ Energy storage Zentralprocessor $\rightarrow$ CPU Datenspreicher $\rightarrow$ Data storage Messwerterfassung $\rightarrow$ Data acquisition Interne Sensoren $\rightarrow$ Internal sensors movement and puls Textile Sensoren (not part of this study b: Schematic /block diagram of the system

very near to the temperature sensor site on the shirt. The movements of the test persons (such as reclining on their left side or standing upright) were documented according to the derivation in the APP, at the same time data was gathered regarding the movements actually observed by the test coordinator.

For phase 2 we followed this plan: in order to investigate the continuous recording or measurement of the parameters, the prototype of the shirt was worn by three test persons over a longer period of between 25 and $50 \mathrm{~min}$ and the data was collected continuously. During the measurement, the test persons recorded abnormalities and the functionality of the shirt and app. Among other things, they tested whether the sensor data were displayed on the app and the data transmission and correct position of the individual sensors in the textile worked in a way that enabled precise measurement results of the vital parameters to be obtained. In the background, the app recorded the continuous sensor data, 


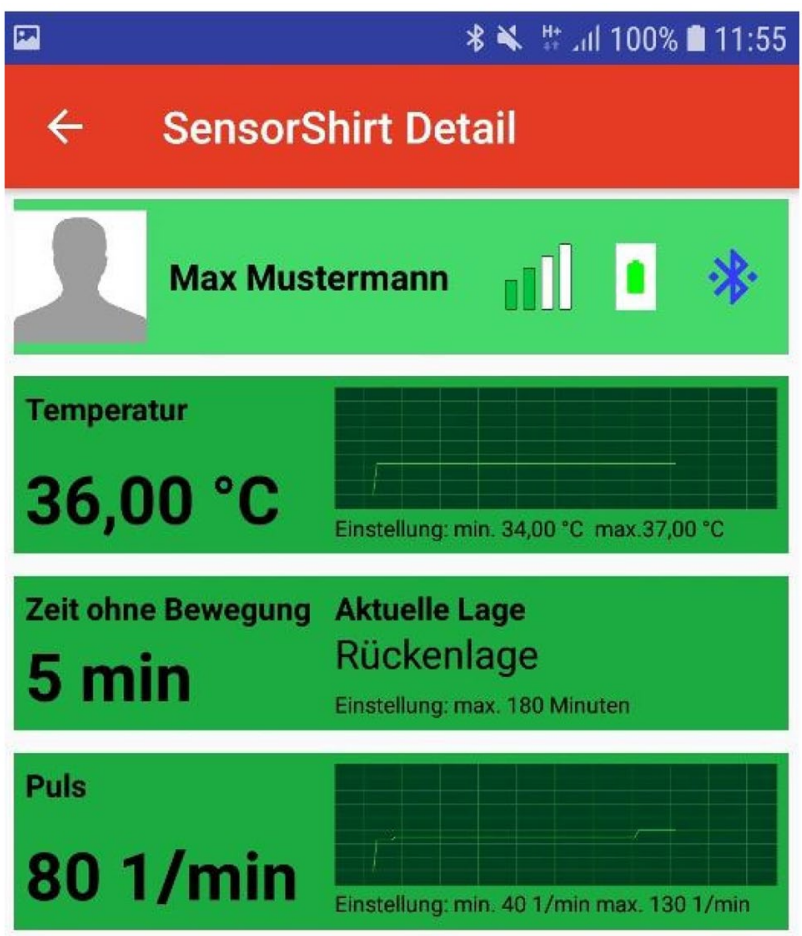

Fig. 5 Multi-Modal Smart Textile APP for android tablets and smartphones (in German) showing the current status of the individual. In the right side under the figures the limits for an alarm are shown. The translation is as follows: Legend (German $\rightarrow$ English): $\bullet$ Temperatur $\rightarrow$ temperature • Einstellung $\rightarrow$ setting $\bullet$ Zeit ohen Bewegung $\rightarrow$ time whithout movement $\bullet$ Aktuelle Lage Rückenlage $\rightarrow$ current position,supine $\bullet$ Pulse $\rightarrow$ pulse

which was stored on the tablet. A function for long-term data storage was implemented. A CSV file was created for each test person to log all vital parameters per second. The measurements and corresponding observations were gathered in a standardised protocol and subsequently fed into to the statistics programme SPSS. The ethics committee of the respective university hospital approved the procedure in March 2018. All participants gave their written informed consent to participate in the study.

\subsubsection{Data analysis}

The data from the two different observations of phase 1 were synchronized in an excel file, the measurements of phase 2 were recorded and all data were analysed using SPSS 24 . For the phase 1 measurements, we compared the two vital signs of pulse and temperature between the two systems. We used the mean values of the measurements per body position to compare the systems. To identify the re-test reliability for measurement, there were repetitions for each system. Interclass correlation coefficients (ICC) with $95 \%$ confidence intervals (CI) were calculated. In order to validate the measurement of the accelerometer, the data of the shirt was compared to the observed body position of the participant. In order to check the match of these two observations, Cohen's kappa is calculated. For phase 2 the data were plotted in line graphs.

\subsection{Subjects}

Five healthy adults participated in the study in phase 1, three of those participated in phase 2. Participants were on average 38.6 years old ( \pm 17.93 years, range: 23 to 67 years). Study phase 1 (phase 2) included four (two) female participants and one (one) male participant. The mean height was $174 \mathrm{~cm}$ (SD 0.15 , range $161-200 \mathrm{~cm}$ ). The regular shirt size of the participants were 2 times small, 2 times large and 1 medium. However, the prototypes were only available in small and medium sizes, so the tests were carried out with these. For phase 1, between 12 and 25 measurements per individual were conducted. For phase 2, the time period in which the individuals were wearing the MMST was $1500 \mathrm{~s}$ ( $25 \mathrm{~min}$ ). All participants gave their written informed consent to participate in the study.
Table 1 Mean values, standard deviation for pulse and temperature measurements of the smart textiles and conventional external measuring devices in comparison $\mathrm{n}=$ number of measurements

\begin{tabular}{|c|c|c|c|c|c|c|c|}
\hline & & smart & & & extern & & \\
\hline & $\begin{array}{l}\text { Position/ } \\
\text { activity }\end{array}$ & mean & sd & $\mathrm{n}$ & Mean & sd & $\mathrm{n}$ \\
\hline pulse & standing & 86.6 & 9.5 & 9 & 80.4 & 20.5 & 9 \\
\hline & sitting & 86.8 & 22.7 & 22 & 71.9 & 12.7 & 22 \\
\hline & lying down & 75.5 & 17.9 & 24 & 67.2 & 10.0 & 24 \\
\hline & moving & 85.1 & 21.8 & 29 & 80.4 & 17.4 & 29 \\
\hline & Total & 82.9 & 20.3 & 84 & 74.4 & 15.6 & 84 \\
\hline temperature & standing & 32.1 & 1.2 & 4 & 31.8 & 0.9 & 4 \\
\hline & sitting & 33.6 & 1.1 & 6 & 32.9 & 1.2 & 6 \\
\hline & lying down & 34.1 & 0.5 & 7 & 31.7 & 1.9 & 7 \\
\hline & moving & 34.6 & 0.5 & 9 & 33.8 & 0.9 & 9 \\
\hline & Total & 33.9 & 1.1 & 26 & 32.7 & 1.6 & 26 \\
\hline
\end{tabular}


Table 2 ICC and Confidence Intervals for the measurements of pulse and temperature

\begin{tabular}{llllll}
\hline & & ICC & $95 \%$ lower ICC & $95 \%$ upper ICC & P-Value \\
\hline pulse & standing & $0.232^{\mathrm{a}}$ & -0.469 & 0.753 & 0.260 \\
& sitting & $0.036^{\mathrm{a}}$ & -0.383 & 0.443 & 0.435 \\
& lying down & $-0.070^{\mathrm{a}}$ & -0.453 & 0.336 & 0.630 \\
& moving & $0.052^{\mathrm{a}}$ & -0.315 & 0.405 & 0.393 \\
\multirow{4}{*}{ temperature } & Total & $0.096^{\mathrm{a}}$ & -0.120 & 0.303 & 0.191 \\
& standing & $0.733^{\mathrm{a}}$ & -0.408 & 0.980 & 0.079 \\
& sitting & $0.817^{\mathrm{a}}$ & 0.162 & 0.972 & 0.012 \\
& lying down & $0.130^{\mathrm{a}}$ & -0.635 & 0.766 & 0.379 \\
& moving & $0.077^{\mathrm{a}}$ & -0.583 & 0.676 & 0.416 \\
& Total & $0.473^{\mathrm{a}}$ & 0.112 & 0.723 & 0.006 \\
\hline
\end{tabular}

\section{Results}

\subsection{Descriptives}

The results of phase 1 are comprised in Table 1 to Table 3 .

Table 1 reports the mean values and standard deviations for mean values between the systems of smart textiles measurement and conventional external measuring devices. Reported mean values of the different measuring instruments differed in a heterogeneous pattern. In particular, the standard deviations of pulse measurements are considered high for the sensor shirt. The number of measurements of the different movement activities (e.g. walking) and different parameters varied. This is due to the fact, that not all of the derived parameters could be measured with the same quality. For instance, the pulse measurements often fluctuated between low and high results when the shirt was worn. Therefore, the measurement of pulse was more frequently than of temperature.

\subsection{Intraclass correlation coefficient (ICC) and Cohen's Kappa}

Table 2 reports the ICC levels of agreement between the measurements of the smart textiles and the conventional clinical devices. The ICC for pulse measurement shows a poor match overall between test and control devices. Moreover, the results are statistically not significant. Overall, the ICC for temperature measurement shows in total a (near to) moderate match between the measurements of the smart textiles and the conventional measurement systems. In particular, the temperature values in a sitting position show a higher level of agreement between smart textiles and the external measuring devices than in other positions. This result is statistically significant. Furthermore, the ICC shows also a high level of agreement in temperature readings for both measurement methods in a standing position. However, this result is not statistically significant.

Table 3 shows a cross-table of observed body positions in comparison to the body positions displayed by the smart textiles. In 39 observations, 34 observations matched in agreement. 5 results show a disagreement in the observation. The table shows a Cohen's Kappa of 0.765 and thus a high interrater reliability and a high match between the results of the observations and the results of the smart textiles. The results are statistically significant.

The results of phase 2 (reliability) are comprised in Fig. 6 a to c, the 3 different colours (blue, red, green) indicate the 3 different individuals who tested the devices.

Figure 6a shows the different pulse values over the course of the examination. The pulse measurements ranged from 60 to 130 beats per minute rapidly. This is a high fluctuation.

Table 3 Cohen's Kappa and cross-table of observed body positions in comparison to smart textiles, key: $1=$ upright body position, $2=$ supine position, $3=$ right reclining position, $4=$ left reclining position

\begin{tabular}{|c|c|c|c|c|c|c|c|c|c|c|c|c|}
\hline & & \multicolumn{10}{|c|}{ body position by observation } & \multirow{3}{*}{$\begin{array}{l}\text { Kappa } \\
\mathrm{p}\end{array}$} \\
\hline & & \multicolumn{2}{|l|}{1} & \multicolumn{2}{|l|}{2} & \multicolumn{2}{|l|}{3} & \multicolumn{2}{|l|}{4} & \multicolumn{2}{|l|}{ Total } & \\
\hline & & $\mathrm{n}$ & $\%$ & $\mathrm{n}$ & $\%$ & $\mathrm{n}$ & $\%$ & $\mathrm{n}$ & $\%$ & $\mathrm{~N}$ & $\%$ & \\
\hline \multirow[t]{4}{*}{ body position by smart textile } & 1 & 23 & $59.0 \%$ & 0 & $0.0 \%$ & 1 & $2.6 \%$ & 0 & $0.0 \%$ & 24 & $61.5 \%$ & \multirow[t]{4}{*}{$0.765(<0.001)$} \\
\hline & 2 & 1 & $2.6 \%$ & 5 & $12.8 \%$ & 0 & $0.0 \%$ & 1 & $2.6 \%$ & 7 & $17.9 \%$ & \\
\hline & 3 & 2 & $5.1 \%$ & 0 & $0.0 \%$ & 3 & $7.7 \%$ & 0 & $0.0 \%$ & 5 & $12.8 \%$ & \\
\hline & 4 & 0 & $0.0 \%$ & 0 & $0.0 \%$ & 0 & $0.0 \%$ & 3 & $7.7 \%$ & 3 & $7.7 \%$ & \\
\hline total & & 26 & $66.7 \%$ & 5 & $12.8 \%$ & 4 & $10.3 \%$ & 4 & $10.3 \%$ & 39 & $100.0 \%$ & \\
\hline
\end{tabular}


Figure $6 \mathrm{~b}$ shows that the given values of the sensor first rise continuously and then remain stable at about $35{ }^{\circ} \mathrm{C}$ on test person $\mathrm{A}$ and $\mathrm{B}$. The fluctuations are smaller for the temperature measurement than for the other measurements of the sensors. Figure $6 \mathrm{c}$ shows the different body positions during testing in phase 2 .

\subsection{Results from the washability testing}

The results of the washing test of the encapsulated electronic module is shown in Table 4. As long as no fabric softener is used, there were no visual abnormities. Full functionality remained after all washings.

\section{Discussion}

The development and testing of a washable multimodal wearable monitoring system contained different steps with heterogeneous results. We were able to integrate the different sensors, microelectronics and technology for data transmission in a washable textile at a reasonable price. The material costs for the prototypes shown here cost about 100 euros. This price could be reduced to half if the quantities are large enough. In this way, we were able to stick goal of developing a system that is as cost-effective as possible and affordable for many people in need of care. The project started in 2016. Although some sources about the washability of E-Textiles were available at that date, we can observe that in the last year a rapid development in this area has occurred. Maxey emphasized that one should not ask whether such a textile is ether washable or not but rather "how much does it degrade after how many cycles and at what setting?" to decide whether or not it is suitable for one purpose [24]. The washability tests were positive. The electronic including the rechargeable NiMH has been washed more than 30 times with different methods and survived these without damage.. The design of a mobile app to show the results was successful. Results were transferred and displayed in a reliable form. The results of the tested parameters pulse, temperature and body position will be discussed in more detail below.

\subsection{Pulse}

In the study, correct derivations of the vital parameter of pulse appeared briefly. In principle, the results indicated an insufficient derivation of this vital function in terms of a valid and reliable measurement. On the one hand it was not possible to achieve a continuous derivation of the pulse parameter using the textile, and on the other hand the match between test and control devices was insufficient. The targeted detection of the pulse on the clavicular artery via two adjacent infrared sensors was possible only to a degree. The sensors were located in the 'technical unit', which was still rather bulky in the prototype, a therefore had a considerable weight (around $75 \mathrm{~g}$ ). As a consequence, the sensors often slipped from their position during the measurement and were sometimes unable to measure the values. This might be less of a problem, if the device becomes lighter and smaller, as this would be our aim in the next step. To our big surprise, that even occurred when the participants were lying down or sitting. Unfortunately, it seems like the optical sensors at this location were very sensitive regarding even lightest movements or even breathing. When the sensor were held tightly in place by pressing it down manually, the reading was correct. Therefore, the textiles should be tailor-made to fit individual wearers and need to fit more snugly. So it can be stated, that for not tight-fitting textiles, a $\mathrm{SpO} 2$ sensor is not a good solution for heart rate measurement. This would require a comprehensive technical and material reworking of the textile, followed by renewed testing to determine whether the pulse parameter can be shown in a valid and reliable manner and can therefore be of use for those concerned and those in need of care. Also alternative technology like the use of Fiber Bragg Grating seems to be promising an should be considered [28].

\subsection{Temperature}

Regarding the parameter of temperature, the results are difficult to interpret. Whilst the results of Fig. $6 \mathrm{~b}$ indicate that the continuous measurement-after some time that the device needs to equilibrate-provides a sufficient level of stability, however the accuracy of this measurement can not be determined due to serious flaws in the methodology that has been applied. In the reviewing process of this manuscript, it became clear, that the laser thermometer that we used was not accurate enough. The results of Table 1 indicate not only that many of the reported mean skin temperatures recorded are lower than would be expected at this site but also that standard deviations of the external device are very high. So why did we use such an external device with such a low accuracy? In fact initially, we ordered a quite accurate laser thermometer for our study but the university administration, addressing economic efficiency, ordered a significant cheaper device with a much lower accuracy. Moreover, an unexperienced research assistant who did not notice the high discrepancies took the measurements with this inaccurate device. Finally, although these measurements started after at least 5 to $10 \mathrm{~min}$, it cannot be guaranteed that the thermometer of the smart shirt had enough time to equilibrate. These potential pitfalls in temperature measurements shows once again the importance of maintaining high scientific standards and quality measures in every step of the research process and must be taken care of in future research. Promising current devolpements are fibers that sense temperature 

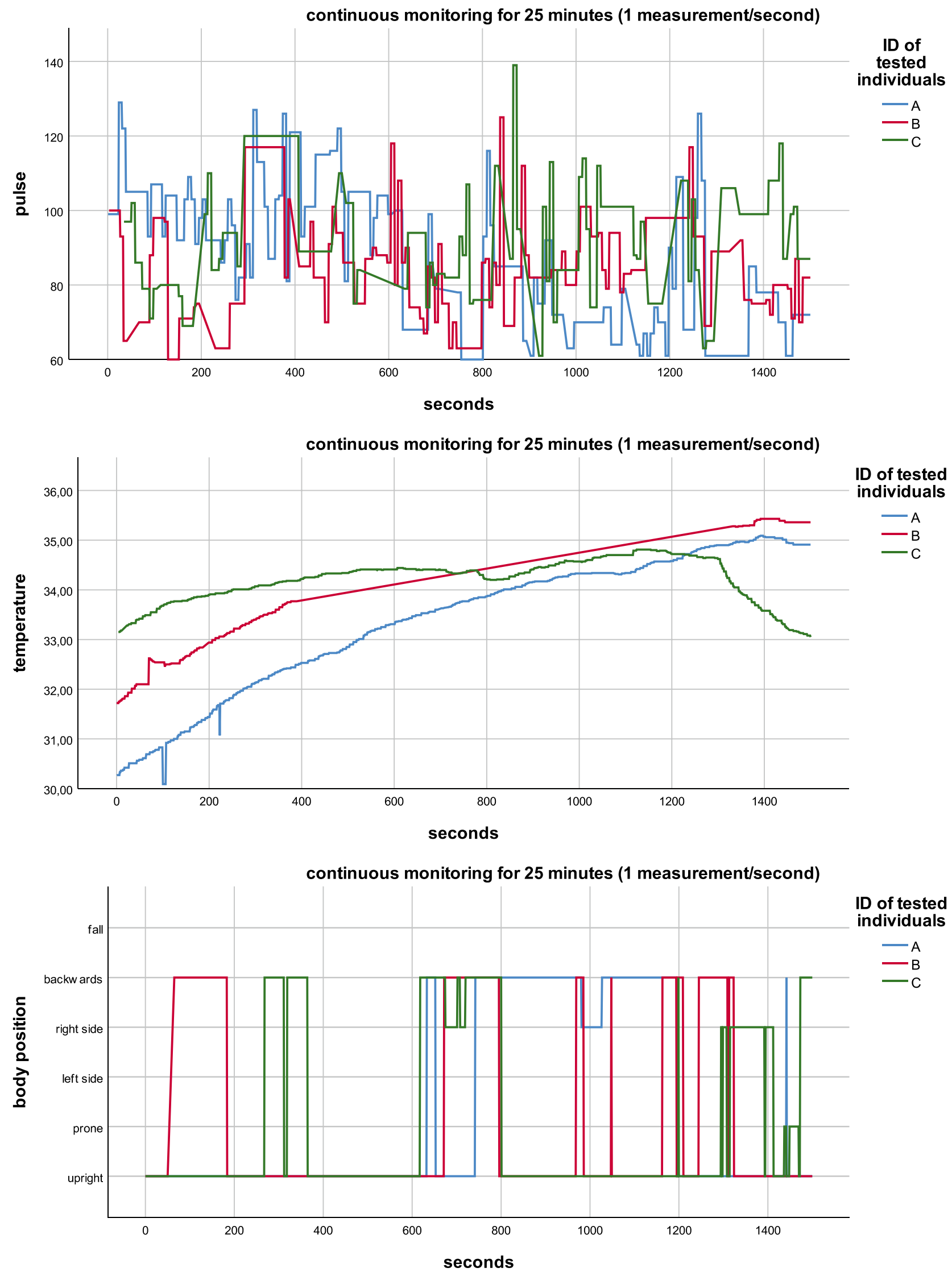

Fig. 6 a: Pulse (BPM) b: Temperature $\left({ }^{\circ}\right.$ Celsius $)$ c: Body Position 
Table 4 Washing tests of the encapsulated electronic module

\begin{tabular}{llllll}
\hline \# of cycles & Washing process & $\begin{array}{l}\text { Duration } \\
(\mathrm{hh}: \mathrm{mm})\end{array}$ & $\begin{array}{l}\text { Spin Speed } \\
(\mathrm{rpm})\end{array}$ & Visual abnormalities & Functionality \\
\hline 1 & without detergent; $40{ }^{\circ} \mathrm{C}$ & $01: 04$ & 400 & none & O.K \\
2 & with detergent \& fabric softener; $40^{\circ} \mathrm{C}$ & $01: 04$ & 400 & silicone slightly torn & O.K \\
3 & without Detergent; $60{ }^{\circ} \mathrm{C}$ & $01: 19$ & 400 & none & O.K \\
4 & with Detergent with $2 \mathrm{~kg}$ laundry; $40{ }^{\circ} \mathrm{C}$ & $01: 04$ & 400 & none & O.K \\
5 & with Detergent with $2 \mathrm{~kg}$ laundry; $95{ }^{\circ} \mathrm{C}$ & $01: 51$ & 1000 & none & O.K \\
$6 .-16$ & $11 \times$ washing with $2 \mathrm{~kg}$ laundry; $40{ }^{\circ} \mathrm{C}$ & $01: 20$ & 800 & none & O.K \\
$17 .-30$ & $14 \times 11 \mathrm{~K}$ washing with $2 \mathrm{~kg}$ laundry; $60^{\circ} \mathrm{C}$ & $01: 30$ & 800 & none & O.K \\
31 & continuously in water & $48: 00$ & - & none &
\end{tabular}

through a thermo-resistive effect [29], but still some challenges in the design of smart materials remain high [30].

The potential lower precision of the measured values has to be considered in the context of costs and intended use. In our case - as we stated in the introduction - it should rather serve as a screening tool. Also, changes in temperature should only be reported in the app, every $10-15 \mathrm{~min}$. With all the measurements in this time period, the microprocessor can provide a reliable mean value, even neglecting outliers. As long as the standard deviations are then normally distributed, a lower accuracy would not really affect the results and the components for the device can remain cheap. In medical context, the core body temperature is usually mostly relevant, but also the temperature of the body surface the data can be considered useful for monitoring care dependent individuals. This allows to detect a temperature rise and enable an appropriately immediate intervention. Another reason is that care dependent people often loose their ability to sense the "right" temperature for themselves, resulting in inadequate dressing themselves. Such a smart textile could help care providers to add are remove clothing in care dependent people if they are are "too cold" or "to hot". The fact that measurements taken in sitting and standing positions show more reliable results indicates or even concludes that the shirt was sitting correctly in these positions and therefore a reliable derivation was possible.

\subsection{Mobility / change of position}

Regarding the mobility parameter, there was a good to sufficient quality of the data regarding validity and reliability. On the one hand, the respective position of the test person (for instance sitting or lying on their left / right side, etc.) and any change of position adopted by the user was recognized and registered on the end-device with a short latency. On the other hand, reliable results were achieved even with repeated measurements. Furthermore, the display of the values was possible in a mostly continuous (without interruption) and error-free manner. The fact that derivation was error-free in a sitting and standing position in particular is probably due to the textile fitting close to the body, which was not the case when lying down on the right or left side of the body. It therefore seems practical to tailor the textiles more individually for a better fit, so the derivation of the mobility or change of position works independently of the position. The parameter mobility / change of position is crucial for preventing bedsores and falls as well as recognizing falls. Fraile et al. point out that the use of non-visible, wearable sensor technology for mobility monitoring in people in need of care can be advantageous [31]. With continuous monitoring available in real time for instance, a fall can be avoided if the sensor triggers an alarm on the respective end device of the relative or carer. Other studies show that the sensor technology applied for mobility data gathering is practicable. Culhane, et al. [32] are of the opinion that long-term mobility observation by use of an accelerometer is a practical instrument in the clinical field. In this case activities such as sitting, standing, lying down and moving could be differentiated by use of the monitoring system [32] in people with limited mobility in particular. It should be noted that by using this and further sensory technologies, a precise, low-cost and easily implemented evaluation of the risk of falls could be achieved. [33]. Although few studies are currently available to provide clinical evidence, Pickham et al. were able to demonstrate that by using wearable sensors, the incidence of pressure ulcers in patients in intensive care could be reduced [34].

Continuous monitoring in real time can protect people in care from bedsores and falls. For example, a fall can be avoided if a sensor triggers an alarm on the corresponding end device of the relative or carer as soon as an immobile person tries to stand up alone. Ultimately it can help to avoid an over- or under provision of care for the patient in the prevention of bedsores. Instead of scheduling a change of position every two or three hours, this can happen as and when it is required. Often, those receiving care still retain a minimal level of remaining mobility, so they are able to move by themselves [35]. A continuously active 
movement sensor can detect this, hence avoiding unnecessary disturbances (of night rest), and relieving care staff and relatives, thus contributing to a higher level of patient safety and quality of care.

\section{Conclusions}

Despite the experienced pitfalls and methodological flaws of our study, we think we were able to show some promising developments that we like to share hereby with the research community. Our used potting compound Wepuran 106 casting resin VT 3402 is solvent-free/VOC-free (Volatile Organic Compounds), has a high mechanical strength, very good protection against impact, shock and vibration and is resistant to water, moisture, condensation water and numerous chemicals, alkalis, acids and oils. However, the manufacturer does not directly guarantee particularly good skin compatibility over a long period of time. We have not carried out long-term tests (longer than 5 days) on the human body. Therefore, future studies may focus on this important safety issue. We are still convinces, that if our washable developed multimodal smart textiles can be successfully modified so that the readings of all vital parameters (pulse, temperature and mobility) deliver reliable results, there would be many advantages for looking after patients who need care, even in an emergency [36]. As a result of this study, it is clear that multiple different sensors have to be used in different body positions in a smart textile. This redundancy can help to detect derivation errors. However, since the correct wearing cannot be guaranteed, it should be considered that the "pulse-rate" should be delivered from another device like a watch or maybe a smart bracelet. Relatively good functionality of the body position detection and the temperature could offer the opportunity to first develop a shirt which offers only this functionality. The advantage would be that such a shirt - if it delivers no vital data - would not be classified as a risk class $2 \mathrm{~b}$ medicinal product. In this case the licence would be much harder to obtain and the final price much higher, making the widespread use of such a system considerably more difficult. Regarding the washability, softener should be avoided, but this is something that many young people may know from other functional fabrics. Since our smart textile addresses rather older individual, adequate care instructions can become very important for washing an e-textile [24].

\subsection{Perspectives}

Although Heo et al. highlighted a couple of challenges in textile-based wearable sensors [37], but considering the fast increase in research and development in this area, it can be expected that this goals will be achievable in the next few years [38-42]. We are aware that the use of other technology like textile-integrated polymer optical fiber sensors would reduce the size and weight of the device and may increase the comfort for those wearing the smart textile. However, an encapsulated microprocessor would still be necessary and also the cost of the whole device would definitely higher. As we learned from our participants, if it is possible to reduce the size of all encapsulated devices down to the size and weight of a small brooth, our technology is quite acceptable. In recent years important progress regarding self-powered platforms in textiles [43], and garments with higher mechanical compliance [44] were reported. However, some of the solutions are still quite expensive and therefore difficult to implement broadly in healthcare. As the sensor could be integrated within the textile, people in care need not be troubled by constantly putting on and taking off the wearable and can be accompanied in a discreet and mainly unnoticeable way though out the day. A modification of the shirt would first be required, with subsequent testing for scientific further development. Along with the optimisation of the technical components, the focus should definitely be on the shirt sitting correctly on the person in care.

In view of the fact that relatives caring for family members are subject to enormous psychological burden and fears, continuous monitoring could raise their sense of security considerably. If the information is processed in a way which is practical and available to all care staff involved (carers, doctors, therapists) this way of feeding back can help in adapting treatment. The increase use of artificial intelligence an machine learning algorithms will help to support this purpose [45]. This is particularly important if the patient has a limited ability to communicate due to dementia. With the implementation of such technology, one must consider that professional as well as informal carers should be trained in the correct use of the clothing. If this is considered, this kind of smart technology not only has a greater chance of acceptance and use in the care of people who need it but could have a great impact on our healthcare system. As Kumari et al. formulated in 2017 in his review of 'Wearable Monitoring Technology": "wearable wireless sensors in which continuous monitoring of patients is possible in real time even without hospitalization. This may be a complete transformation of existing healthcare system" [46].

Acknowledgements We would like to thank Nicola Varns for the English proofreading of this manuscript.

Author contributions Conceptualization: N.A.L., S.K., J.K.-N., U.M.-W., F.J., S.S.-L.; funding acquisition: N.A.L., S.K., and U.M.W methodology: N.A.L., S.K. and S.S.-L.; software: F.J. formal analysis: N.A.L. and S.S.-L.; writing — original draft: N.A.L., J.K.-N., S.S.-L.; writing-review and editing: N.A.L., S.K., J.K.-N., U.M.-W., 
F.J., S.S.-L.; visualization: N.A.L.,F.J., S.K. and S.S.-L.; supervision: S.S.-L. All authors have read and agreed to the published version of the manuscript.

Funding Open Access funding enabled and organized by Projekt DEAL. The project was a governmental funded cooperation between two academic institutions and 2 SMEs. Projectleader for the Charité was N.A.L. The Funding was a part of a governmental support program, the so-called "Central Innovation Programme for small and medium-sized enterprises (SMEs)" and was sponsored by the Federal Ministry for Economic Affairs and Energy (funding reference number: ZF4288601SU6). Freyer \& Siegel provided support in the form of salaries for the author Fabian Jaenicke, but did not have any additional role in the study design, data collection and analysis, decision to publish, or preparation of the manuscript. The specific roles of these authors are articulated in the 'author contributions' section.

Data availability All data could be sent to the journal for critical evaluation.

\section{Declarations}

Ethical approval The ethics committee of the Charite-Universitätsmediz in Berlin approved the procedure and informed consent of this research in March 2018 (EA2/046/18). All participants gave their written informed consent to participate in the study.

Conflict of interests The authors declare the supporting sources were not involved in the decision to submit the report for publication.

Open Access This article is licensed under a Creative Commons Attribution 4.0 International License, which permits use, sharing, adaptation, distribution and reproduction in any medium or format, as long as you give appropriate credit to the original author(s) and the source, provide a link to the Creative Commons licence, and indicate if changes were made. The images or other third party material in this article are included in the article's Creative Commons licence, unless indicated otherwise in a credit line to the material. If material is not included in the article's Creative Commons licence and your intended use is not permitted by statutory regulation or exceeds the permitted use, you will need to obtain permission directly from the copyright holder. To view a copy of this licence, visit http://creativecommons.org/licenses/by/4.0/.

\section{References}

1. Lauterbach C, Glaser R, Savio D, Schnell M, Weber W. Integrated microelectronics for smart textiles. Stud Health Technol Inform. 2005;117:72-9.

2. Van Langenhove L, Hertleer C, Catrysse M, Puers R, Van Egmond H, Matthijs D. Smart textiles. Stud Health Technol Inform. 2004;108:344-52.

3. Burns M. Medical Trauma Assessment Through the Use of Smart Textiles. In Sci M Eng Inc, Ed. 1995.

4. Park S, Gopalsamy C, Rajamanickam R, Jayaraman S. The Wearable Motherboard: a flexible information infrastructure or sensate liner for medical applications. Stud Health Technol Inform. 1999;62:252-8.

5. Lee D, Sang JS, Yoo PJ, Shin TJ, Oh KW, Park J. Machine-Washable Smart Textiles with Photothermal and Antibacterial Activities from Nanocomposite Fibers of Conjugated Polymer Nanoparticles and Polyacrylonitrile. Polymers (Basel). 2018;11(1).
6. Stular D, Jerman I, Naglic I, Simoncic B, Tomsic B. Embedment of silver into temperature- and $\mathrm{pH}$-responsive microgel for the development of smart textiles with simultaneous moisture management and controlled antimicrobial activities. Carbohydr Polym. 2017;159:161-70.

7. Weng W, Chen P, He S, Sun X, Peng H. Smart Electronic Textiles. Angew Chem Int Ed Engl. 2016;55(21):6140-69.

8. Liu C, Liu F, Zhang L, Su Y, Murray A. Smart Wearables in Healthcare: Signal Processing, Device Development, and Clinical Applications. J Healthc Eng. 2018;2018:1696924.

9. Davies N. WEARABLES, IMPLANTS AND APPS, Oh My! SMART TECHNOLOGY FOR DIABETES SELF-CARE. Diabetes Self Manag. 2016;33(2):28-31.

10. Yan W. Toward Better Management for Asthma: From Smart Inhalers to Injections to Wearables, Researchers Are Finding New Ways to Improve Asthma Treatment. IEEE Pulse. 2018;9(1):28-33.

11. Ko J, Kim NH. Evolution of Smart Health Wearables: Novel Application for Detection of Arrhythmia. Korean Circ J. 2019;49(5):446-7.

12. Laguna Sanz AJ, Diez JL, Gimenez M, Bondia J. Enhanced Accuracy of Continuous Glucose Monitoring during Exercise through Physical Activity Tracking Integration. Sensors (Basel). 2019;19(17).

13. Smiley A, Majerus SJA, McAdams IS, Hanzlicek B, Bourbeau D, Damaser MS. Sensors Selection for Continuous Monitoring of Bowel State and Activity. Conf Proc IEEE Eng Med Biol Soc. 2018;2018:2997-3000.

14. Al Sayed C, Vinches L, Hallé S. Validation of a Wearable Biometric System's Ability to Monitor Heart Rate in Two Different Climate Conditions under Variable Physical Activities. E-Health Telecommunication Systems and Networks. 2017;6:19-30.

15. Bruce-Low SS, Cotterrell D, Jones GE. Effect of wearing personal protective clothing and self-contained breathing apparatus on heart rate, temperature and oxygen consumption during stepping exercise and live fire training exercises. Ergonomics. 2007;50(1):80-98.

16. Feito Y, Moriarty TA, Mangine G, Monahan J. The use of a smarttextile garment during high-intensity functional training: a pilot study. J Sports Med Phys Fitness. 2019;59(6):947-54.

17. Tao X, Koncar V, Huang TH, Shen CL, Ko YC, Jou GT. How to Make Reliable, Washable, and Wearable Textronic Devices. Sensors (Basel). 2017;17(4).

18. Najafi B, Mohseni H, Grewal GS, Talal TK, Menzies RA, Armstrong DG. An Optical-Fiber-Based Smart Textile (Smart Socks) to Manage Biomechanical Risk Factors Associated With Diabetic Foot Amputation. J Diabetes Sci Technol. 2017;11(4):668-77.

19. Koyama Y, Nishiyama M, Watanabe K. Smart Textile Using Hetero-Core Optical Fiber for Heartbeat and Respiration Monitoring. IEEE Sens J. 2018;18(15):6175-80.

20. Massaroni C, Saccomandi P, Schena E. Medical smart textiles based on fiber optic technology: an overview. J Funct Biomater. 2015;6(2):204-21.

21. Presti DL, Massaroni C, D’Abbraccio J, Massari L, Caponero M, Longo UG, Formica D, Oddo CM, Schena E. Wearable System Based on Flexible FBG for Respiratory and Cardiac Monitoring. IEEE Sensors J. 2019;19(17):7391-7398.

22. Zhou Z, Padgett S, Cai Z, Conta G, Wu Y, He Q, Zhang S, Sun C, Liu J, Fan E, Meng K, Lin Z, Uy C, Yang J, Chen J. Single-layered ultra-soft washable smart textiles for all-around ballistocardiograph, respiration, and posture monitoring during sleep. Biosens Bioelectron. 2020;155:112064.

23. Ghosh S, Nitin B, Remanan S, Bhattacharjee Y, Ghorai A, Dey T, Das TK, Das NC. A Multifunctional Smart Textile Derived from Merino Wool/Nylon Polymer Nanocomposites as Next Generation 
Microwave Absorber and Soft Touch Sensor. ACS Appl Mater Interfaces. 2020;12(15):17988-8001.

24. Maxey M. Smart Fabrics are Coming, But What About Washability and E-textiles Degradation? https://fashnerd.com/2019/ 06/e-textiles-washability-degradation-future-of-fashion/ (Nov. 6),

25. Afroj S, Karim N, Wang Z, Tan S, He P, Holwill M, Ghazaryan D, Fernando A, Novoselov KS. Engineering Graphene Flakes for Wearable Textile Sensors via Highly Scalable and Ultrafast Yarn Dyeing Technique. ACS Nano. 2019;13(4):3847-57.

26. Liu Q, Huang J, Zhang J, Hong Y, Wan Y, Wang Q, Gong M, Wu Z, Guo CF. Thermal, Waterproof, Breathable, and Antibacterial Cloth with a Nanoporous Structure. ACS Appl Mater Interfaces. 2018;10(2):2026-32.

27. Vervoust T, Buyle G, Bossuyl F, Vanfleteren J. Integration of stretchable and washable electronic modules for smart textile applications. J Text Inst. 2012;103(10):1127-38.

28. Lo Presti D, Romano C, Massaroni C, D’Abbraccio J, Massari L, Caponero MA, Oddo CM, Formica D, Schena E. Cardio-respiratory monitoring in archery using a smart textile based on flexible fiber Bragg grating sensors. Sensors. 2019;19(16):3581.

29. Lake G, Alain J, Yan W, Khudiyev T, Noel G, Yuan R, Missakian A, Fink Y. Computing Fabrics Matter. 2020;2:786-8.

30. Yan W, Dong C, Xiang Y, Jiang S, Leber A, Loke G, Xu W, Hou C, Zhou S, Chen M, Hu R, Shum PP, Wei L, Jia X, Sorin F, Tao X, Tao G. Thermally drawn advanced functional fibers: New frontier of flexible electronics. Mater Today. 2020;35:168-94.

31. Fraile JA, Bajo J, Corchado JM, Abraham A. Applying wearable solutions in dependent environments. IEEE Trans Inf Technol Biomed. 2010;14(6):1459-67.

32. Culhane KM, Lyons GM, Hilton D, Grace PA, Lyons D. Longterm mobility monitoring of older adults using accelerometers in a clinical environment. Clin Rehabil. 2004;18(3):335-43.

33. Sun R, Sosnoff JJ. Novel sensing technology in fall risk assessment in older adults: a systematic review. BMC geriatrics. 201818(1):1-0

34. Pickham D, Berte N, Pihulic M, Valdez A, Mayer B, Desai M. Effect of a wearable patient sensor on care delivery for preventing pressure injuries in acutely ill adults: A pragmatic randomized clinical trial (LS-HAPI study). Int J Nurs Stud. 2018;80:12-9.

35. Carrea-Bassin F, Cotte S, Junuzi S, Bouchard D, Thomas P. Using new technology to enable preventing pressure ulcers and falls in a University Hospital in Switzerland - a clinical projet. Belfast 2017.
36. Rebeccah PF. Electronics and fabrics: the development of garment based wearables. Adv Mater Technol. 2018;3:1-6.

37. Heo JS, Hossain MF, Kim I. Challenges in design and fabrication of flexible/stretchable carbon-and textile-based wearable sensors for health monitoring: A critical review. Sensors. 2020;20(14):3927.

38. Dong C, Leber A, Gupta TD, Chandran R, Volpi M, Qu Y, Nguyen-Dang T, Bartolomei N, Yan W, Sorin F. High-efficiency super-elastic liquid metal based triboelectric fibers and textiles. Nat comm. 2020;11(1):1-9.

39. Loke G, Yan W, Khudiyev T, Noel G, Fink Y. Recent progress and perspectives of thermally drawn multimaterial fiber electronics. Adv Mater 2020;32(1):1904911.

40. Shi J, Liu S, Zhang L, Yang B, Shu L, Yang Y, Ren M, Wang Y, Chen J, Chen W, Chai Y. Smart textile-integrated microelectronic systems for wearable applications. Adv maters. 2020;32(5): 1901958.

41. Yan W, Richard I, Kurtuldu G, James ND, Schiavone G, Squair JW, Nguyen-Dang T, Das Gupta T, Qu Y, Cao JD, Ignatans R, Lacour SP, Tileli V, Courtine G, Loffler JF, Sorin F. Structured nanoscale metallic glass fibres with extreme aspect ratios. Nat Nanotechnol. 2020;15(10):875-82.

42. Wicaksono I, Tucker CI, Sun T, Guerrero CA, Liu C, Woo WM, Pence EJ, Dagdeviren C. A tailored, electronic textile conformable suit for large-scale spatiotemporal physiological sensing in vivo. npj Flexible Electron. 2020;4(1):1-3.

43. Jeerapan I, Sempionatto JR, Pavinatto A, You JM, Wang J. Stretchable Biofuel Cells as Wearable Textile-based Self-Powered Sensors. J Mater Chem A Mater. 2016;4(47):18342-53.

44. Nazmul Islam GM, Azam A, Stewart C. Textile sensors for wearable applications: a comprehensive review. Cellulose. 2020;27:6103-31.

45. Vu CC, Kim J. Human motion recognition by textile sensors based on machine learning algorithms. Sensors. 2018;18(9):3109.

46. Kumari P, Lopez-Benitez M, Gyu Myoung L, Tae-Seong K, Minhas AS. Wearable Internet of Things - from human activity tracking to clinical integration. Conf Proc IEEE Eng Med Biol Soc. 2017;2017:2361-4.

Publisher's Note Springer Nature remains neutral with regard to jurisdictional claims in published maps and institutional affiliations. 